Proceedings

\title{
Evaluation of the Hypolipidemic Properties of Cocoa Shell after Simulated Digestion Using In Vitro Techniques and a Cell Culture Model of Non-Alcoholic Fatty Liver Disease ${ }^{+}$
}

\author{
Cheyenne Braojos 1,2, , Vanesa Benitez 1,2, Miguel Rebollo-Hernanz 1,2, , Silvia Cañas 1,2, Yolanda Aguilera 1,2, \\ Silvia M. Arribas ${ }^{3}$ and Maria A. Martin-Cabrejas ${ }^{1,2}$
}

Citation: Braojos, C.; Benitez, V.; Rebollo-Hernanz, M.; Cañas, S.; Aguilera, Y.; Arribas, S.M.; MartinCabrejas, M.A. Evaluation of the Hypolipidemic Properties of Cocoa Shell after Simulated Digestion Using In Vitro Techniques and a Cell Culture Model of Non-Alcoholic Fatty Liver Disease. Proceedings 2021, 70, 58. https://doi.org/10.3390/ foods_2020-07669

Published: 9 November 2020

Publisher's Note: MDPI stays neutral with regard to jurisdictional claims in published maps and institutional affiliations.

Copyright: () 2020 by the authors. Licensee MDPI, Basel, Switzerland. This article is an open access article distributed under the terms and conditions of the Creative Commons Attribution (CC BY) license (http://creativecommons.org/licenses/by/4.0/).
1 Department of Agricultural Chemistry and Food Science, Universidad Autónoma de Madrid, 28049 Madrid, Spain; vanesa.benietz@uam.es (V.B.); silvia.cannas@uam.es (S.C.); yolanda.aguilera@uam.es (Y.A.); maria.martin@uam.es (M.A.M.C.)

2 Institute of Food Science Research, CIAL (UAM-CSIC), 28049 Madrid, Spain

3 Department of Physiology, Universidad Autónoma de Madrid, 28029 Madrid, Spain; silvia.arribas@uam.es

* Correspondence: cheyenne.braojos@uam.es (C.B.); miguel.rebollo@uam.es (M.R.-H.)

† Presented at the 1st International Electronic Conference on Food Science and Functional Foods, 10-25 November 2020. Available online: https://foods_2020.sciforum.net/.

\begin{abstract}
Obesity is closely associated with the increasing prevalence of non-alcoholic fatty liver disease (NAFLD). Due to the lack of proper pharmacological treatments for NAFLD, finding novel ingredients is necessary to reduce its incidence. Cocoa shell is a cocoa byproduct verified as a safe ingredient and a potential source of health-promoting compounds. Hence, this study's main objective was to evaluate, after an in vitro simulated digestion, the hypolipidemic properties of the residual fraction of cocoa shell flour and the biological activity of the digested fractions of cocoa shell flour and extract in HepG2 cells. An in vitro static digestion (INFOGEST) of cocoa shell flour was used to establish the residual fraction's capacity to bind cholesterol and bile salts and inhibit lipase. The results showed that digestion promoted the ability to bind cholesterol and bile salts of a residual fraction from a cocoa shell up to $65.2 \%$ and $90.5 \%$. Moreover, digestion improved (1.6-fold, $p<0.05$ ) the ability to inhibit lipase activity. The digested fractions of the flour and extract from the cocoa shell $(50-250 \mu \mathrm{g} / \mathrm{mL})$ significantly $(p<0.05)$ reduced the accumulation of fat $(17-42 \%)$, triglycerides (9-38\%), and cholesterol (11-54\%) in HepG2 cells after NAFLD induction with palmitic acid (500 $\mu \mathrm{M})$. In conclusion, digestion positively impacted the hypolipidemic properties of cocoa shells in vitro and enhanced their biological activity in cell culture models. Since cocoa shells might be used as a safe, novel ingredient to prevent hyperlipidemia and regulate lipid metabolism, future animal and clinical investigations will be necessary to confirm the effects observed.
\end{abstract}

Keywords: cocoa by-products; cocoa shell; gastrointestinal digestion; hypolipidemic; hypocholesterolemic; non-alcoholic fatty liver disease

\section{Introduction}

Obesity is defined as an excessive accumulation of adipose tissue. It is closely associated with multiple metabolic risk factors for a cardiovascular disease, including insulin resistance, diabetes, dyslipidemia, and a spectrum of liver abnormalities, known as nonalcoholic fatty liver disease (NAFLD) [1]. NAFLD has become one of the leading causes of chronic liver disease, representing the hepatic manifestation of the metabolic syndrome, ranging from simple steatosis (i.e., fat accumulation in more than $5 \%$ of the hepatocytes) to necroinflammation and fibrosis, leading to non-alcoholic steatohepatitis (NASH), which often results in a progression to cirrhosis, liver failure, and hepatocellular 
carcinoma [2,3]. Many pharmacological treatments have been proposed for the management of NAFLD. However, none of them is approved in clinical use currently [4]. Due to the lack of proper pharmacological treatments for NAFLD, modifying the diet to prevent obesity and its comorbidities is the principal candidate. In this context, finding novel ingredients that may help us to reduce the incidence of these disorders is necessary.

During cocoa bean processing, approximately $90 \%$ is discarded as cocoa byproducts, which have a high potential for developing new products. The cocoa shell is the tegument that covers the cocoa bean. It is obtained during the cocoa bean roasting process, constituting about $10-17 \%$ of the total cocoa bean weight [5]. The cocoa shell is a rich source of carbohydrates (62\%) and proteins (10-27\%), as well as dietary fiber (DF) (59\%) and valuable bioactive compounds (theobromine, caffeine, phenolic compounds, etc.), which can be used as an ingredient in the food industry, among other industries [6,7]. Furthermore, cocoa shells have recently been validated as a potential safe ingredient. It is a potential source of nutrients and health-promoting compounds used as nutraceuticals to manage chronic diseases [7]. Phytochemicals from cocoa shells have been associated with the regulation of biomarkers of metabolic syndrome (lipid accumulation, inflammation, oxidative stress, and insulin resistance) [8]. Therefore, this study's main objective was to evaluate, after an in vitro simulated digestion, the hypolipidemic properties of the residual fraction of cocoa shell flour and the biological activity of the digested fractions of cocoa shell flour and extract in HepG2 cells.

\section{Materials and Methods}

\subsection{Materials}

Dulbecco's Modified Eagle's Medium low glucose (1000 mg/mL) (DMEM) and 0.25\% trypsin-EDTA were purchased from GE Healthcare Life S Life Technologies. [3-(4,5-dimethylthiazol-2-yl)-5-(3-carboxymethoxyphenyl)-2-(4-sulfophenyl)-2H-tetrazolium (MTS) was purchased from Promega Corporation (Carlsbad, CA, USA). $o$-Phthalaldehyde, furfural, bile salts, lipase, Tween ${ }^{\circledR}$ 20, Bovine Serum Albumin (BSA), Palmitic Acid (PA), 2', $7^{\prime}$ dichlorofluorescein diacetate (DCFDA), and Oil Red O were purchased from Sigma-Aldrich (St. Louis, MO, USA). All other chemicals and reagents were obtained from Panreac (Barcelona, Spain), unless otherwise specified.

\subsection{Cocoa Flour and Aqueous Extracts Preparation}

The cocoa shell was provided by Chocolates Santocildes (Castrocontrigo, León, Spain). Cocoa shell flour was obtained after milling and cocoa shell aqueous extracts described by Rebollo-Hernanz et al. [9].

\subsection{INFOGEST Static In Vitro Simulation Digestion}

Digestions were carried out based on the preliminary and optimized protocols described by Brodkorb et al. [10]

\subsection{Cholesterol-Binding Capacity}

For the measure of the residue's cholesterol-binding capacity, fresh egg yolks were diluted 1:10 $(v / w)$, and the dilution was emulsified, divided, and adjusted to $\mathrm{pH} 2.0$ and $\mathrm{pH}$ 7.0. Samples $(0.1 \mathrm{~g})$ were mixed with the diluted egg yolk $(2 \mathrm{~mL})$ and incubated with agitation $\left(2 \mathrm{~h}, 37^{\circ} \mathrm{C}\right)$, followed by an $800 \mathrm{~g}$ centrifugation ( $\left.15 \mathrm{~min}\right)$. Consecutively, samples supernatants $(0.1 \mathrm{~mL})$ were mixed with $0.6 \mathrm{~mL}$ of pure acetic acid, $0.2 \mathrm{~mL}^{\circ} \mathrm{H}_{2} \mathrm{SO}_{4}(96 \%)$, and $0.1 \mathrm{~mL}$ of $o$-phthalaldehyde $(0.6 \mathrm{mg} / \mathrm{mL})$ and incubated while mixing for color development $\left(30 \mathrm{~min}, 60^{\circ} \mathrm{C}\right)$. Finally, triplicate-measured absorbance at $550 \mathrm{~nm}$ using a microplate reader (Cytation 5, Biotek). 


\subsection{Bile Salts-Binding Capacity}

A mixture of $\mathrm{NaCl}(15 \mathrm{M}, \mathrm{pH} 7.0)$ and sodium cholate $(4.65 \mathrm{mM})$ was added to $0.1 \mathrm{~g}$ of the residual fraction of gastric and intestinal flour digestion. The samples were incubated for $3 \mathrm{~h}$ at $37^{\circ} \mathrm{C}$ while mixing, followed by an $800 \times g$ 15-min centrifugation. Hereafter, $0.6 \mathrm{~mL}$ of $\mathrm{H}_{2} \mathrm{SO}_{4}(45 \%)$ and $0.1 \mathrm{~mL}$ of furfural $(0.3 \%)$ were added to $0.1 \mathrm{~mL}$ of the supernatant. The samples were incubated with oscillations for color development (30 min, $65^{\circ} \mathrm{C}$ ). Absorbance was measured by triplicate at $620 \mathrm{~nm}$ using a microplate reader.

\subsection{Inhibitory Activity against Pancreatic Lipase}

To determine the inhibitory activity against pancreatic lipase, a mixture consisting of olive oil $(2 \mathrm{~mL})$, lipase $(2 \mathrm{~mL}, 0.75 \mathrm{mg} / \mathrm{mL})$, bile salts $(5 \mathrm{~mL})$, and phosphate-buffered saline (PBS) $10 \times(5 \mathrm{~mL}, \mathrm{pH} 7.2)$ was added to $0.1 \mathrm{~g}$ of the residual fraction of each flour digestion. Controls were carried with or without each compound (including the residual fraction). Samples were incubated $\left(37^{\circ} \mathrm{C}, 1 \mathrm{~h}\right)$. The reaction was stopped in the cold for 5 $\mathrm{min}$, followed by centrifugation $(800 \times g, 15 \mathrm{~min})$. Hereafter, Tween ${ }^{\circledR} 20(0.2 \mathrm{~mL})$ was added to the supernatant of those samples that contained olive oil to emulsify the fats. Finally, each sample's lipase activity was measured using an acid-base titration with $\mathrm{NaOH}(0.02$ M) and phenolphthalein.

\subsection{Treatment Preparation for HepG2 Cells}

The digested fractions of cocoa shell flour (CSF) and extract (CSE) were diluted in DMEM-free and filtered with $0.45-\mu \mathrm{m}$ cellulose acetate filters.

\subsection{Cell Culture Method}

HepG2 cells were obtained from ATCC (Rockville, MD, USA). Cells were cultured in DMEM (1000 g/mL glucose) supplemented with $10 \%$ FBS and $1 \%$ penicillin/streptomycin at $37^{\circ} \mathrm{C}$ in a humidified atmosphere.

\subsubsection{Cell Viability}

HepG2 cells were seeded at $5 \times 10^{5}$ cells $/ \mathrm{mL}$ in 96-well culture plates and incubated for $24 \mathrm{~h}$. On day 2, cells were treated with CSF and CSE $(50-250 \mathrm{mg} / \mathrm{mL})$ for $24 \mathrm{~h}$ at $37^{\circ} \mathrm{C}$ in a humidified atmosphere. On day 3 , a cell viability assay was performed with CellTiter $96^{\circledR}$ AQueous MTS Reagent Powder according to the manufacturer's instructions.

\subsubsection{NAFLD Induction in HepG2 Cells}

HepG2 were seeded at $10^{5}$ cells $/ \mathrm{cm}^{2}$ in 96-well culture plates and incubated for $24 \mathrm{~h}$. On day 2, NAFLD was induced in HepG2 cells with PA $(500 \mu \mathrm{M})$, and they were treated with CSF or CSE $(100 \mu \mathrm{g} / \mathrm{mL})$ for $24 \mathrm{~h}$ at $37^{\circ} \mathrm{C}$ in a humidified atmosphere. To prepare the PA solution, PA was conjugated with DMEM containing 1\% BSA. Control cells were treated with $1 \%$ BSA only.

\subsubsection{Reactive Oxygen Species Formation in HepG2 Cells}

After 24-h incubation with treatments, NAFLD induction was determined by measuring the intracellular reactive oxygen species (ROS) formation by adding DCFDA ( 25 $\mu \mathrm{M})$. Fluorescence intensity was detected using a fluorescence microplate reader at an excitation/emission wavelength of $485 \mathrm{~nm} / 530 \mathrm{~nm}$, respectively. DCFDA was prepared according to the manufacturer's instructions.

\subsubsection{Biological Hypolipidemic Activity}

The accumulation of total lipids and intracellular triglycerides was performed as previously described by Rebollo-Hernanz et al. [9] using Oil Red O and a colorimetric triglycerides assay kit (Spinreact), respectively, according to the manufacturer's instructions. 
Cholesterol accumulation was measured as previously described by Ryu et al. [11] using a colorimetric cholesterol assay kit (Spinreact) according to the manufacturer's instructions.

\subsection{Statistical Analysis}

All experiments were carried out in triplicate. Results were expressed as the mean \pm standard deviation (SD) $(n=3)$ and were assessed statistically by one-way analysis of variance (ANOVA) and post hoc Tukey's test. Differences were defined as statistically significant for values of $p<0.05$. All analyses were performed using SPSS 24.0.

\section{Results}

\subsection{Simulated In Vitro Digestion Enhanced the Hypolipidemic Properties of Cocoa Shell}

As observed in Figure 1A, the digestion process affected the cholesterol-binding capacity in the cocoa shell. Simulating the physiological parameters, gastric digestion residues at $\mathrm{pH} 2$ and gastrointestinal phase at $\mathrm{pH} 7$, reduced the cholesterol-binding capacity. However, it improved during colonic digestion at both $\mathrm{pH}$. On the other hand, the digestion of cocoa shell significantly $(p<0.05)$ improved bile salts-binding capacity during all digestion phases (Figure 1B). Furthermore, cocoa shell digestion positively enhanced the lipase inhibition during the gastric and gastrointestinal phases with or without the bile salts addition (Figure 1C).

A

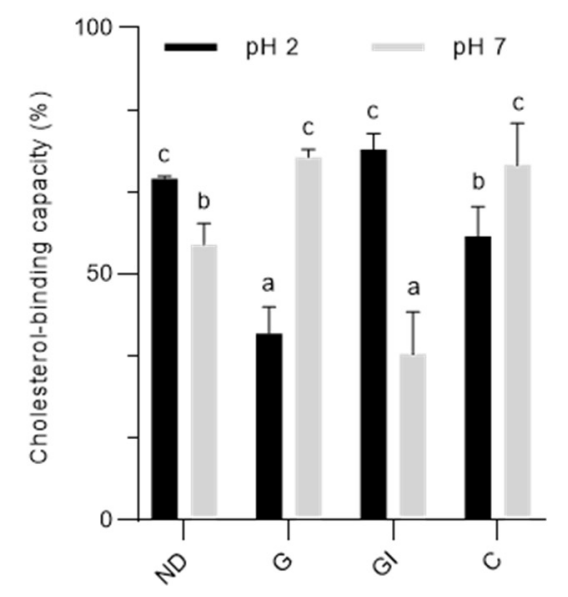

B

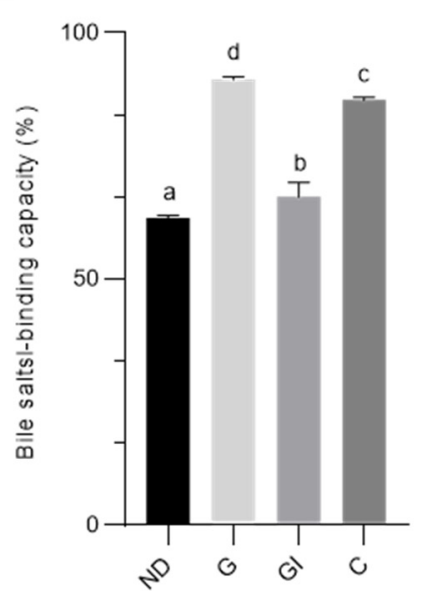

C

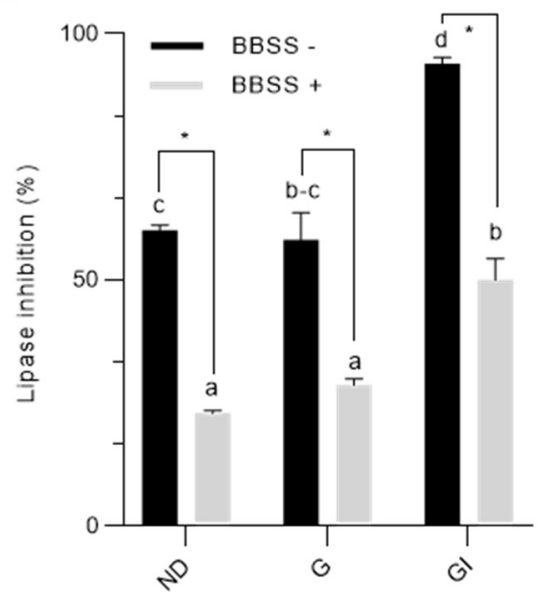

Figure 1. Effects of digestion on the cocoa shell hypolipidemic properties. (A) Cholesterol-binding capacity of cocoa shell digested residual fractions at $\mathrm{pH} 2.0$ and $\mathrm{pH}$ 7.0. (B) Bile salts-binding capacity of cocoa shell digested residual fractions. (C) In vitro lipase inhibition capacity of cocoa shell digested residual fractions without bile salts (BBSS-) and containing them (BBSS+). The results are expressed as the mean \pm SD. Bars with different letters significantly $(p<0.05)$ differ according to ANOVA and Tukey's multiple range test. Statistically significant $(p<0.05)$ differences between paired samples according to the $t$-test are represented by an asterisk $\left.{ }^{*}\right)$. ND: nondigested flour, G: gastric residual fraction, GI: intestinal residual fraction, and C: colonic residual fraction.

\subsection{Cocoa Shell Was Not Toxic and Regulated PA-Stimulated ROS Formation and Lipid Accumulation}

To simulate the NAFLD model on cell cultures, HepG2 cells were incubated for $24 \mathrm{~h}$ with PA $(500 \mu \mathrm{M})$, and the ROS levels were measured to study the NAFLD induction. An MTS assay was carried out to determine the effects of different concentrations of CSE and CSF $(50-250 \mu \mathrm{g} / \mathrm{mL})$ on HepG2 proliferation, and the findings indicated that all the concentrations used were not cytotoxic and significantly $(p<0.05)$ reduced the toxic effects of PA. The results showed that PA significantly $(p<0.05)$ induced ROS formation. Moreover, 
the HepG2 cell morphology changed after incubation with PA, favoring the formation of lipid drops (Figure 2). CSE and CSF $(50-250 \mu \mathrm{g} / \mathrm{mL})$ significantly $(p<0.05)$ reduced the accumulation of fat (17-42\%) and modulated the triglyceride and cholesterol levels, lowering them by about $9-38 \%$ and $11-54 \%(p<0.05)$, respectively, after the induction of NAFLD with PA.
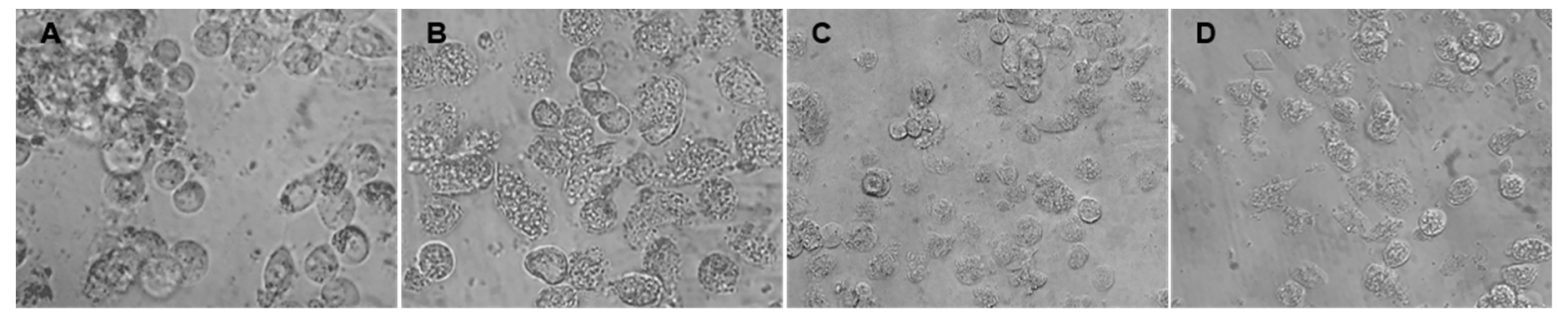

Figure 2. Illustrative representation of HepG2 morphologies after incubation in different conditions. (A) Cells cultured in Dulbecco's Modified Eagle's Medium-Bovine Serum Albumin (DMEM-BSA) 1\%. (B) Cells cultured in DMEM-BSA 1\% with Palmitic Acid (PA) $(500 \mu \mathrm{M})$. (C) Cells cultured in DMEM-BSA $1 \%$ with PA $(500 \mu \mathrm{M})$ treated with gastric cocoa shell extract (CSE) $(100 \mu \mathrm{g} / \mathrm{mL})$. (D) Cells cultured in DMEM-BSA 1\% with PA (500 $\mu \mathrm{M})$ treated with gastric CSE $(200 \mu \mathrm{g} / \mathrm{mL})$.

\section{Discussion}

Cocoa has been reported to have benefits for health [12]. The cocoa shell composition has been described to be similar to that of cocoa beans. It also presents hypolipidemic and hypoglycemic properties in vitro, which may be explained because of its chemical composition [5,7]. Cocoa shells are a rich source of DF [7]. DF is not absorbed nor digested during the human gastrointestinal digestion process but could be wholly or partially fermented [13]. DF has been reported to mediate lipid metabolism and cholesterol absorption. Previous studies have demonstrated that most NAFLD patients follow a diet poor in DF $[14,15]$. The residual fraction of the simulated gastric and gastrointestinal digestion of cocoa shell flour showed less cholesterol-binding capacity at physiological $\mathrm{pH}$ (2.0 and 7.0, respectively) than nondigested flour. Besides, the residual fraction of every phasesimulated digestion improved the bile salts-binding capacity. Previous studies have demonstrated that DF possesses a bile salts-binding ability and modulates the accumulation of circulating triglycerides by inhibiting the bile salt surfactant activity, which reduces micelle formations during intestinal digestion and, therefore, intestinal lipid absorption [16]. Likewise, DF can reduce cholesterol absorption. The residual fraction of every simulated digestion of cocoa shell flour showed higher pancreatic lipase inhibition than nondigested flour. The DF bile salts-binding capacity reduces micelle formation, explaining the decrease in lipase activity because of a reduction in lipid accessibility. It could also explain why bile salts added to the lipase inhibition method reduced the lipase inhibition capacity of cocoa shell flour [16]. Similarly, DF can entrap the enzyme (lipase) or the substrate (triglycerides) and reduce its activity [17]. These results suggest that cocoa shell digestion could enhance its hypolipidemic properties, which could play an essential role in treating or preventing chronic diseases associated with hyperlipidemia, such as NAFLD.

Studies in HepG2 cells showed that CSE and CSF reduced the lipid, cholesterol, and triglycerides accumulation after the induction of simulated NAFLD and could significantly reduce ROS levels, probably due to the antioxidant properties of the cocoa shell polyphenols [18]. Some authors suggested that oxidative stress and mitochondrial dysfunction could be critical factors during NAFLD progression [19]. Previous studies in our group proved the effects of cocoa shell phytochemicals on protecting mitochondrial function and regulating metabolism in hepatocytes [20]. The results obtained suggest the potential role of the cocoa shell in preventing this disease. From this perspective, and based on the high DF and phenolic compound contents of cocoa shell, evaluating the effects of its digestion on the hypolipidemic properties and biological activity is necessary to reassign them as novel ingredients that could have health benefits in some diseases like NAFLD and could also have a positive impact on the environment. Further investigations 
on the bio-accessible and the nondigestible fractions of the cocoa shell and their in vivo functional properties should be completed to confirm its health properties.

\section{Conclusions}

Digestion positively impacted the cocoa shell's hypolipidemic properties, leading to enhanced biological activity in vitro and in cell culture models. Since the cocoa shell might be used as a safe, novel ingredient to prevent hyperlipidemia and regulate lipid metabolism, future animal and clinical investigations will be necessary to confirm the effects observed in vitro.

Author Contributions: Conceptualization, M.R.H., V.B., Y.A., and M.A.M.C; formal analysis, C.B. and M.R.H.; investigation, C.B.; data curation, C.B. and S.C.; writing-original draft preparation, C.B., M.R.H., and V.B.; writing - review and editing, C.B., M.R.H., and V.B.; supervision, V.B. and M.A.M.C; and funding acquisition, S.M.A. and M.A.M.C. All authors have read and agreed to the published version of the manuscript.

Funding: This research was funded by the COCARDIOLAC project from the Spanish Ministry of Science and Innovation (RTI 2018-097504-B-I00). M. Rebollo-Hernanz received funding from the FPU program of the Ministry of Science, Innovation, and Universities for his predoctoral fellowship (FPU15/04238).

Institutional Review Board Statement: Not applicable.

Informed Consent Statement: Not applicable.

Data Availability Statement: The data presented in this study are available on request from the corresponding author on reasonable request.

Conflicts of Interest: The authors declare no conflicts of interest.

\section{References}

1. Fabbrini, E.; Sullivan, S.; Klein, S. Obesity and nonalcoholic fatty liver disease: Biochemical, metabolic, and clinical implications. Hepatology 2010, 51, 679-689, doi:10.1002/hep.23280.

2. Demir, M.; Lang, S.; Steffen, H.M. Nonalcoholic fatty liver disease-Current status and future directions. J. Dig. Dis. 2015, 16, 541-557, doi:10.1111/1751-2980.12291.

3. Matteoni, C.A.; Younossi, Z.M.; Gramlich, T.; Boparai, N.; Yao Chang Liu; McCullough, A.J. Nonalcoholic fatty liver disease: A spectrum of clinical and pathological severity. Gastroenterology 1999, 116, 1413-1419, doi:10.1016/S0016-5085(99)70506-8.

4. Lau, L.H.S.; Wong, S.H. Microbiota, obesity and NAFLD. Adv. Exp. Med. Biol. 2018, 1061, 111-125, doi:10.1007/978-981-10-8684-7_9.

5. Rojo-Poveda, O.; Barbosa-Pereira, L.; Zeppa, G.; Stévigny, C. Cocoa bean shell-A by-product with nutritional properties and biofunctional potential. Nutrients 2020, 12, 1123, doi:10.3390/nu12041123.

6. Panak Balentić, J.; Ačkar, Đ.; Jokić, S.; Jozinović, A.; Babić, J.; Miličević, B.; Šubarić, D.; Pavlović, N. Cocoa Shell: A By-Product with Great Potential for Wide Application. Molecules 2018, 23, 1404, doi:10.3390/molecules23061404.

7. Rebollo-Hernanz, M.; Cañas, S.; Aguilera, Y.; Benitez, V.; Gila-Díaz, A.; Rodriguez-Rodriguez, P.; Cobeta, I.M.; de Pablo, A.L.L.; Gonzalez, M.C.; Arribas, S.M.; et al. Validation of Cocoa Shell as a Novel Antioxidant Dietary Fiber Food Ingredient: Nutritional Value, Functional Properties, and Safety. Curr. Dev. Nutr. 2020, 4, 773-773, doi:10.1093/cdn/nzaa052_042.

8. Rebollo-Hernanz, M.; Zhang, Q.; Aguilera, Y.; Martín-Cabrejas, M.A.; de Mejia, E.G. Relationship of the phytochemicals from coffee and cocoa by-products with their potential to modulate biomarkers of metabolic syndrome in vitro. Antioxidants 2019, 8 , 279, doi: 10.3390/antiox8080279.

9. Rebollo-Hernanz, M.; Zhang, Q.; Aguilera, Y.; Martín-Cabrejas, M.A.; Gonzalez de Mejia, E. Phenolic compounds from coffee by-products modulate adipogenesis-related inflammation, mitochondrial dysfunction, and insulin resistance in adipocytes, via insulin/PI3K/AKT signaling pathways. Food Chem. Toxicol. 2019, 132, 110672, doi:10.1016/j.fct.2019.110672.

10. Brodkorb, A.; Egger, L.; Alminger, M.; Alvito, P.; Assunção, R.; Ballance, S.; Bohn, T.; Bourlieu-Lacanal, C.; Boutrou, R.; Carrière, F.; et al. INFOGEST static in vitro simulation of gastrointestinal food digestion. Nat. Protoc. 2019, 14, 991-1014, doi:10.1038/s41596-018-0119-1.

11. Ryu, H.M.; Kim, Y.J.; Oh, E.J.; Oh, S.H.; Choi, J.Y.; Cho, J.H.; Kim, C.D.; Park, S.H.; Kim, Y.L. Hypoxanthine induces cholesterol accumulation and incites atherosclerosis in apolipoprotein E-deficient mice and cells. J. Cell. Mol. Med. 2016, 20, 2160-2172, doi:10.1111/jcmm.12916.

12. Badrie, N.; Bekele, F.; Sikora, E.; Sikora, M. Cocoa Agronomy, Quality, Nutritional, and Health Aspects. Crit. Rev. Food Sci. Nutr. 2015, 55, 620-659, doi:10.1080/10408398.2012.669428. 
13. Macagnan, F.T.; da Silva, L.P.; Hecktheuer, L.H. Dietary fibre: The scientific search for an ideal definition and methodology of analysis, and its physiological importance as a carrier of bioactive compounds. Food Res. Int. 2016, 85, 144-154, doi:10.1016/j.foodres.2016.04.032.

14. Hong, Y.; Zi-jun, W.; Jian, X.; Ying-jie, D.; Fang, M. Development of the dietary fiber functional food and studies on its toxicological and physiologic properties. Food Chem. Toxicol. 2012, 50, 3367-3374, doi:10.1016/j.fct.2012.05.011.

15. Eslamparast, T.; Tandon, P.; Raman, M. Dietary Composition Independent of Weight Loss in the Management of Non-Alcoholic Fatty Liver Disease. Nutrients 2017, 9, 800, doi:10.3390/nu9080800.

16. Capuano, E. The behavior of dietary fiber in the gastrointestinal tract determines its physiological effect. Crit. Rev. Food Sci. Nutr. 2017, 57, 3543-3564, doi:10.1080/10408398.2016.1180501.

17. Benítez, V.; Rebollo-Hernanz, M.; Aguilera, Y.; Bejerano, S.; Cañas, S.; Martín-Cabrejas, M.A. Extruded coffee parchment shows enhanced antioxidant, hypoglycaemic, and hypolipidemic properties by releasing phenolic compounds from the fibre matrix. Food Funct. 2021, 12, 1097, doi: 10.1039/D0FO02295K.

18. Rebollo-Hernanz, M.; Zhang, Q.; Aguilera, Y.; Martín-Cabrejas, M.A.; de Mejia, E.G. Cocoa shell aqueous phenolic extract preserves mitochondrial function and insulin sensitivity by attenuating inflammation between macrophages and adipocytes in vitro. Mol. Nutr. Food Res. 2019, 63, 1801413, doi: 10.1002/mnfr.201801413.

19. Fontes, A.; Alemany-Pagès, M.; Oliveira, P.J.; Ramalho-Santos, J.; Zischka, H.; Azul, A.M. Antioxidant versus pro-apoptotic effects of mushroom-enriched diets on mitochondria in liver disease. Int. J. Mol. Sci. 2019, 20, 3987, doi:10.3390/ijms20163987.

20. Rebollo-Hernanz, M.; Willis, L.; Aguilera, Y.; Martin-Cabrejas, M.A.; Gonzalez de Mejia, E. Fibroblast Growth Factor 21 Signaling Activation by Selected Bioactive Compounds from Cocoa Shell Modulated Metabolism and Mitochondrial Function in Hepatocytes. Curr. Dev. Nutr. 2020, 4, 459-459, doi: 10.1093/cdn/nzaa045_092. 